\title{
Integrated optimization of control surface layout for gust load alleviation
}

\author{
Manuel Pusch ${ }^{1}$ (I) Andreas Knoblach ${ }^{1} \cdot$ Thiemo Kier $^{1}$
}

Received: 24 July 2018 / Revised: 21 December 2018 / Accepted: 8 February 2019 / Published online: 13 February 2019

(C) The Author(s) 2019

\begin{abstract}
Considering active gust load alleviation (GLA) during aircraft design offers great potential for structural weight savings. The effectiveness of a GLA control system strongly depends on the layout of available control surfaces, which is investigated in this article. For the purpose of wing load reduction, a concurrent optimization of controller gains and aileron geometry parameters is carried out. To that end, an efficient update routine for the nonlinear model of a large-scale flexible aircraft with unsteady aerodynamics is presented. Compared to a GLA system using the original aileron configuration, a 9\% performance improvement is achieved. Furthermore, a trade-off study is carried out which enables a target-oriented balancing between individual load channels. The significant influence of aileron size and position on overall GLA performance is demonstrated and hence a consideration during the preliminary aircraft design process is recommended.
\end{abstract}

Keywords Multidisciplinary design optimization $\cdot$ Control surface design $\cdot$ Gust load alleviation $\cdot$ Aeroservoelasticity

\section{Introduction}

To allow for a more economic and environmentally friendly operation of aircraft, fuel savings are imperative. Besides the efficiency of engines and aerodynamics, the aircraft weight has a major impact on fuel consumption [1]. For instance, a reduction of aircraft weight is achieved using new materials like carbon composites, as it can be seen at the example of the Airbus A350 or the Boeing 787. Another approach is to decrease the design loads of the structure [2, 3] applying active control technologies. For example, the fuel consumption of the Lockheed L-1011 TriStar aircraft could be reduced by $3 \%$ by means of active load alleviation [4]. Considering new aircraft configurations with improved lift-to-drag ratios, a special focus has to be put on gust load alleviation (GLA), as these configurations are prone to have an increased sensitivity to atmospheric disturbances. In [5], an assessment of state of the art GLA applications is made and its potential for weight reductions is pointed out. In industry though, advanced load alleviation functions are still often introduced after the preliminary design phase [5-7],

Manuel Pusch

manuel.pusch@dlr.de

1 Institute of System Dynamics and Control, German Aerospace Center, 82234 Weßling, Germany where only a limited adaption of the structure is possible. Hence, it is advantageous to include the load alleviation system as early as possible in the aircraft design cycle [8]. Promising results are achieved by multidisciplinary design optimization, where aircraft structure and load controller are designed simultaneously (see e.g. $[9,10]$ ). However, less priority is put on optimization of the layout of multifunctional control surfaces and its concrete impact on load alleviation capability.

The aim of this paper is to investigate the potential of simultaneously optimizing the GLA controller gains and the respective control surface layout. To gain realistic results, a flexible aircraft model of industrial complexity is considered in Sect. 2. The nonlinear model includes unsteady aerodynamics and allows to compute cut loads for maneuvers as well as gust encounters. In avoidance of time-consuming model re-building, an efficient update procedure for control surface layout changes is proposed. In the derived optimization setup (Sect. 3), the focus lies on simultaneously optimizing controller and aileron geometry parameters to minimize the wing root bending moment. Additionally, constraints like actuator saturation, passenger comfort and stability requirements are considered. The resulting improvement in load alleviation capability is discussed in Sect. 4, where the optimized aileron layout is compared with a reference configuration. Finally, 
a trade-off study is carried out to allow a globally balanced load reduction by prioritizing single load channels.

\section{Modeling and loads computation}

\subsection{Structural and aerodynamic model}

To consider both gust and maneuver loads, the integrated modeling approach from [11] is applied. The model is based on a linear finite element model on which a modal analysis is carried out. The resulting mode shapes are partitioned into rigid body modes $\boldsymbol{\Phi}_{\mathrm{gb}}$ and flexible modes $\boldsymbol{\Phi}_{\mathrm{gf}}$. Taking into account the assumptions from [12], this allows to replace the linear rigid body dynamics by the nonlinear equations of motion (EoM) from flight mechanics. Eventually, the overall EoM can be written as

$\left[\begin{array}{c}m_{\mathrm{b}}\left(\dot{\mathbf{V}}_{\mathrm{b}}+\dot{\boldsymbol{\Omega}}_{\mathrm{b}} \times \mathbf{V}_{\mathrm{b}}-\mathbf{T}_{\mathrm{bE}} \mathbf{g}_{\mathrm{E}}\right) \\ \mathbf{J}_{\mathrm{b}} \dot{\boldsymbol{\Omega}}_{\mathrm{b}}+\mathbf{\Omega}_{\mathrm{b}} \times\left(\mathbf{J}_{\mathrm{b}} \boldsymbol{\Omega}_{\mathrm{b}}\right)\end{array}\right]=\boldsymbol{\Phi}_{\mathrm{gb}}^{\mathrm{T}} \mathbf{P}_{\mathrm{g}}^{\text {ext }}(t)$,

$\mathbf{M}_{\mathrm{ff}} \ddot{u}_{\mathrm{f}}+\mathbf{B}_{\mathrm{ff}} \dot{\mathbf{u}}_{\mathrm{f}}+\mathbf{K}_{\mathrm{ff}} \mathbf{u}_{\mathrm{f}}=\boldsymbol{\Phi}_{\mathrm{gf}}^{\mathrm{T}} \mathbf{P}_{\mathrm{g}}^{\text {ext }}(t)$.

In Eq. (1), the rigid body modes are described in the body frame of reference by the translational velocity $\mathbf{V}_{b}$ and the angular velocity $\boldsymbol{\Omega}_{\mathrm{b}}$. Additionally, gravitational acceleration $\mathbf{g}_{\mathrm{E}}$ is taken into account by applying a coordinate transformation $\mathbf{T}_{\mathrm{bE}}$ from the earth fixed to the body fixed frame. It is further assumed that $\mathbf{g}_{\mathrm{E}}$ as well as the inertia tensor $\mathbf{J}_{\mathrm{b}}$ and the aircraft mass $m_{\mathrm{b}}$ do not change within the considered time horizon. In Eq. (2), the flexible modes $\mathbf{u}_{\mathrm{f}}$ are characterized by means of the modal mass matrix $\mathbf{M}_{\mathrm{ff}}$, the modal damping matrix $\mathbf{B}_{\mathrm{ff}}$ and the modal stiffness matrix $\mathbf{K}_{\mathrm{ff}}$.

The external nodal loads $\mathbf{P}_{\mathrm{g}}$ ext include forces induced by aerodynamics, engines or landing gears. For the purpose of GLA, aerodynamic forces are of major interest. To consider also unsteady aerodynamics, they are obtained by means of the doublet lattice method (DLM) [13]. Applying the DLM, the lifting surfaces are discretized by trapezoidal shaped aerodynamic boxes with a control point $j$ located at the three quarter chord respectively (see Fig. 1).

Fig. 1 Aerodynamic box of chord length $c_{j}$ with reference point $k$ and control point $j$
The orthogonal components of the flow at these control points are collected in $\mathbf{v}_{j}$ and normalized by the free stream velocity $U_{\infty}$, leading to the downwash

$\mathbf{w}_{j}=\frac{\mathbf{v}_{j}}{U_{\infty}}$.

As a result of the DLM, the aerodynamic loads acting on the nodes of the structural model are given as

$\mathbf{P}_{g}^{\text {aero }}=\mathbf{Q}_{g j}(k) \mathbf{w}_{j}$,

where the aerodynamic influence coefficient (AIC) matrix $\mathbf{Q}_{g j}(k)$ is typically computed only at discrete reduced frequencies $k$ [13]. To enable time domain simulations, a rational function approximation (RFA) of $\mathbf{Q}_{g j}(k)$ is derived using Roger's method [14]. According to Eq. (3), the aerodynamic loads depend linearly on the downwash $\mathbf{w}_{j}$, which consists of a gust-, modal- and control surface (CS)-component. For the gust downwash, the continuous wind field is evaluated at each aerodynamic box and the respective orthogonal components are normalized by the free stream velocity. And the other two downwash components result from the movements of aerodynamic boxes caused by modal displacements and CS deflections, respectively. Note that the translations and rotations of aerodynamic boxes are generally described with respect to the midpoint $k$ of each box (see also Fig. 1) and hence, a transformation to the control point $j$ is necessary. A more detailed explanation on downwash computation is given in [11] and in the next subsection, where the model updating procedure for changing the CS layout is described. Furthermore, it has to be mentioned that the aerodynamic model depends on the current Mach number, air density and free stream velocity, see also $[11,13]$ for details.

Eventually, the nodal loads $\mathbf{P}_{\mathrm{g}}$ are recovered using the force summation method (FSM) [15]:

$\mathbf{P}_{\mathrm{g}}=\mathbf{P}_{\mathrm{g}}^{\text {ext }}-\mathbf{P}_{\mathrm{g}}^{\text {iner }}$,

where the nodal inertial loads $\mathbf{P}_{g}^{\text {iner }}$ are obtained from the accelerations of the rigid body and flexible modes. In comparison to that, the mode displacement method (MDM) [15] computes the nodal loads by

$\mathbf{P}_{\mathrm{g}}=\mathbf{K}_{\mathrm{gg}} \boldsymbol{\Phi}_{\mathrm{gf}} \mathbf{u}_{\mathrm{f}}$,

using the physical stiffness matrix $\mathbf{K}_{\mathrm{gg}}$. Generally, the MDM exhibits an inferior convergence behavior [15] and thus, it is not applied here. However, for interpretation of the results, the MDM is useful as it allows to determine the contributions of the corresponding flexible modes to the overall loads. Finally, the integrated cut loads $\mathbf{P}_{\mathrm{c}}$ at critical cross sections, e.g. the wing root, are computed by a linear transformation which sums up the corresponding nodal loads. 


\subsection{Control surfaces}

In general, the downwash $\mathbf{w}_{j}^{\mathrm{x}}$ caused by CS deflections $\mathbf{u}_{x}$ is described by

$\mathbf{w}_{j}^{\mathrm{x}}=\left(\mathbf{D}_{j k}^{1}+s \frac{c_{\mathrm{ref}} / 2}{U_{\infty}} \mathbf{D}_{j k}^{2}\right) \boldsymbol{\Phi}_{k x} \mathbf{u}_{x}$,

where the CS matrix $\boldsymbol{\Phi}_{k x}$ maps the CS deflections to the movement of individual aerodynamic boxes. The mapping depends on the relative position of the box to the respective CS hinge and is computed applying a small-angle approximation. The differentiation matrices $\mathbf{D}_{j k}^{1}$ and $\mathbf{D}_{j k}^{2}$ are introduced in order to transform the box displacements and movements from the reference point $k$ to a downwash at the control point $j$ (see also [11]). Besides, the Laplace variable is denoted by $s$ and the reference chord length of the aircraft is $c_{\text {ref }}$.

When changing the geometry of a CS, it is necessary to rebuild the underlying aerodynamic lattice to align it with the new boundaries of the modified CS. This, in turn, requires the AIC matrix to be recomputed and approximated again by a rational function. To avoid this rather time-consuming process during optimization, an alternative approach is proposed here. The AIC matrix is computed only once and the aerodynamic lattice is not further modified. Instead, the present aerodynamic boxes are assigned to the current CSs in a proportional manner.

Giving an example, the size of the original CS from Fig. 2a (covered by box 4 and 6) is meant to be decreased. In Fig. 2b, the aerodynamic lattice is updated, leading to a new set of aerodynamic boxes requiring the AIC to be recomputed. In comparison to that, in Fig. $2 \mathrm{c}$ the aerodynamic lattice is not changed but the box assignments are weighted individually. For box 4 , this implies that it is weighted by a factor of 0.6 as it is covered by the new CS only by $60 \%$. Similarly, box 6 is weighted by $100 \%$ meaning that it is fully assigned to the CS.

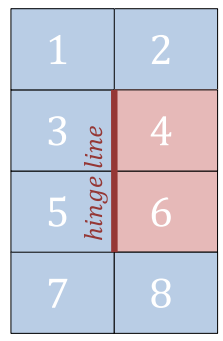

(a) Original

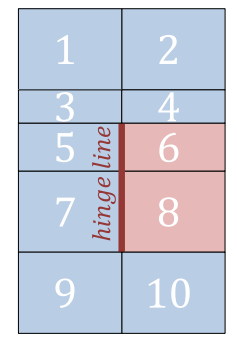

(b) Updated

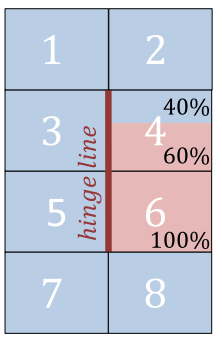

(c) Weighted
Fig. 2 Example of changing the size of a CS where the updated aerodynamic panels are compared to the original aerodynamic panels with updated weights
In summary, each box is weighted according to the percentage of its area overlapping with the respective CS. Thus, only the entries of $\boldsymbol{\Phi}_{k x}$ related to the modified CSs need to be updated, whereas the rest of the aircraft model remains unchanged. As the mass distribution and stiffness are assumed not to be influenced, the emerging approximation error is negligible for sufficiently small aerodynamic boxes.

\subsection{Actuators and sensors}

The actuator dynamics of the CSs are modeled by a first order low pass filter

$G_{\mathrm{acts}}(s)=\frac{\omega_{\mathrm{c}}}{s+\omega_{\mathrm{c}}}$,

with a bandwidth $\omega_{\mathrm{c}}=20 \mathrm{rad} \mathrm{s}^{-1}$. For an active GLA at the wing, the inner and outer ailerons are primarily used as they can be deflected in both directions which allows controlling the wing lift distribution effectively. In addition to that, the elevators are used to compensate the pitching moment induced by aileron deflections. Furthermore, the sensor signals for feedback control are the measured pitch rate $q_{\text {meas }}$ and the measured vertical acceleration $a_{z \text {,meas }}$ from the inertial measurement unit (IMU), which is located close to the aircraft center of gravity (CoG). Both signals are readily available in common aircraft and thus, no extra sensors need to be added.

\subsection{Limit loads computation}

To size the structure of an aircraft, it is necessary to determine the limit loads. According to the certification requirements [2,3], the limit loads are the lower and upper boundary of all loads occurring during aircraft operation at any time. Denoted as $\mathbf{P}_{\mathrm{c} \text {,lower }}$ and $\mathbf{P}_{\mathrm{c} \text {,upper }}$, the limit loads are determined in this paper by simulating extreme flight maneuvers and severe atmospheric turbulence as described in the following subsections.

\subsubsection{Maneuver limit loads}

In Table 1, trim conditions for representative steady flight maneuvers, used to determine the maneuver limit loads, are listed. At each flight point, the steady horizontal flight M0 with zero pitch rate $q$ and zero roll rate $p$ is trimmed through the horizontal stabilizer. Additionally, the push-over maneuver M1 a and the pull-up maneuver M1b are performed. Both maneuvers are trimmed by means of elevator deflections $\eta$ and differ from each other only by the load factor $n_{z}$. The load factors $n_{z, \min }$ and $n_{z, \text { max }}$ are specified in the flight maneuvering envelope $(V-n$ diagram) [2,3] and depend on the design airspeed. Similarly, the bidirectional rolling maneuvers M3a and M3b are trimmed 
Table 1 Trim table of maneuvers to compute limit loads

\begin{tabular}{lllllllll}
\hline ID & Maneuver name & $n_{z}$ & $p$ & $\dot{p}$ & $q$ & $\dot{q}$ & $\eta$ & $\xi$ \\
\hline M0 & Horizontal flight & 1 & 0 & 0 & 0 & 0 & 0 & 0 \\
M1a & Push-over & $n_{z, \min }$ & 0 & 0 & $?$ & 0 & $?$ & 0 \\
M1b & Pull-up & $n_{z, \max }$ & 0 & 0 & $?$ & 0 & $?$ & 0 \\
M2a & Pilot pull & $?$ & 0 & 0 & $?$ & $?$ & $\eta(\mathrm{M} 1 \mathrm{a})$ & 0 \\
M2b & Pilot push & $?$ & 0 & 0 & $?$ & $?$ & $\eta(\mathrm{M} 1 \mathrm{~b})$ & 0 \\
M3a & Roll and pull & 0 & $\pm p_{\max }$ & 0 & $?$ & 0 & $?$ & $?$ \\
M3b & Roll and push & $\frac{2}{3} n_{z, \max }$ & $\pm p_{\max }$ & 0 & $?$ & 0 & $?$ & $?$ \\
M4a & Pilot roll and pull & 0 & 0 & $?$ & $?$ & 0 & $?$ & $\pm \xi(\mathrm{M} 3 \mathrm{a})$ \\
M4b & Pilot roll and push & $\frac{2}{3} n_{z, \max }$ & 0 & $?$ & $?$ & 0 & $?$ & $\pm \xi(\mathrm{M} 3 \mathrm{~b})$ \\
\hline
\end{tabular}

by means of the aileron deflections $\xi$. Moreover, sudden pilot commands are approximated by the accelerated roll maneuvers M4a and M4b, and the accelerated pitching maneuvers $\mathrm{M} 2 \mathrm{a}$ and $\mathrm{M} 2 \mathrm{~b}$. The extreme pilot inputs are determined by the CS deflections resulting from the previous maneuvers and are assumed to be established instantly.

By definition, the maximum roll rate $p_{\max }$ is set to $15^{\circ} \mathrm{s}^{-1}$ for all operation points, which is a common value for civil aircraft. Furthermore, for all maneuvers, inner and outer ailerons are deflected equally but with opposite sign on the left and right wing. In contrast, elevators are always deflected symmetrically.

\subsubsection{Gust limit loads}

To compute the structural loads in atmospheric turbulence, the "1-cos" gust model according to the certification requirements $[2,3]$ is used. For wing loads, gusts in up- and downwards direction are considered as the most critical. Thus, time domain simulations are carried out for vertical gusts with different gust gradient distances $H$ varying from $9 \mathrm{~m}$ (30 ft) to $107 \mathrm{~m}(350 \mathrm{ft})$.

\section{Optimization setup}

\subsection{Controller structure}

For active GLA, a multiple-input multiple-output controller needs to be designed using the sensor and actuator signals described in Sect. 2.3. Since a concurrent optimization of the controller and the control surface layout is carried out, a minimum number of controller tuning parameters is desired allowing for a smooth and fast convergence. Based on that and the findings of [16], a static gain feedback controller is chosen, where high frequency modes are not excited due to the low-pass behavior of the actuators. As only symmetrical gusts in vertical direction are considered (see Sect. 2.4), the CS deflection commands are applied equally on the left- and right-hand side. Thus, the controller outputs are $\Delta \eta_{\mathrm{cmd}}$ for the elevators, $\Delta \xi_{\text {inner,cmd }}$ for the inner ailerons and $\Delta \xi_{\text {outer,cmd }}$ for outer ailerons. Introducing the static gain feedback matrix $\mathbf{K}$, the controller structure is given as

$\left[\begin{array}{c}\Delta \eta_{\mathrm{cmd}} \\ \Delta \xi_{\text {inner,cmd }} \\ \Delta \xi_{\text {inner,cmd }}\end{array}\right]=\mathbf{K}\left[\begin{array}{c}\Delta a_{z \text {,meas }} \\ \Delta q_{\text {meas }}\end{array}\right]$

where all signals represent increments (denoted by the $\Delta$ ) with respect to the current trim conditions M0 given in Table 1. For a smooth controller tuning, the input and output signals of the controller are normalized by their respective maximum values. The scaled elements of $\mathbf{K}$ are then collected in the controller tuner parameter vector $\mathbf{D}_{K}$ used for optimization (see Sect. 3.5).

\subsection{Parameterization of ailerons}

To evaluate the impact of the aileron layout on GLA performance, the geometry of the ailerons is parameterized. In Fig. 3, different parameterizations of the span-wise position and the span of the inner and outer ailerons are given. The parameter space is limited by the minimum and maximum aileron position $y_{\min }$ and $y_{\max }$. While the minimum position is defined by the planform break, the maximum position is the outer boundary of the reference aileron configuration as depicted in Fig. 7. The ailerons should not be placed further outside as the trailing vortex at the wing tip may cause unfavorable effects [17]. For ailerons optimization,

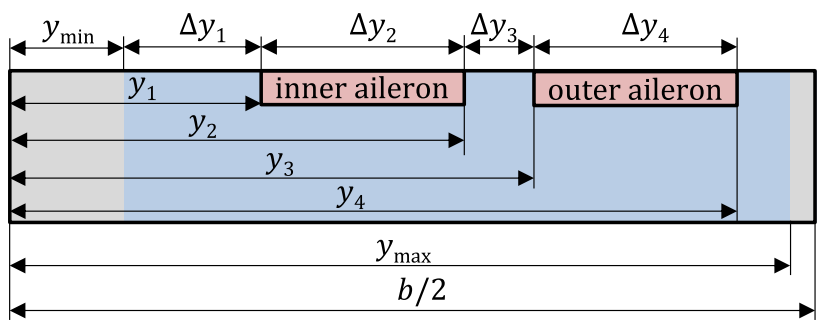

Fig. 3 Ailerons parameterization 


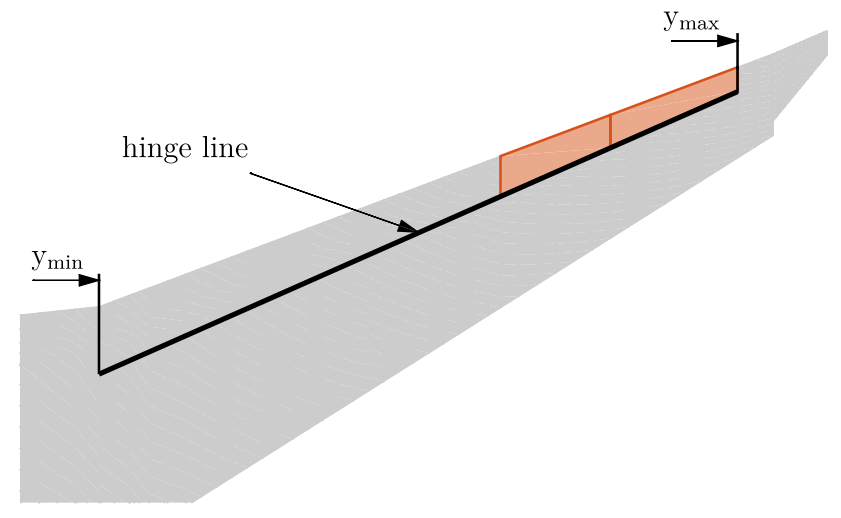

Fig. 4 Reference aileron layout with limits for layout optimization

three different parameter sets $\mathbf{D}_{\text {ail }}$ are tested: (1) the absolute positions $y_{1} \cdots y_{4}$, (2) the distances $\Delta y_{1} \cdots \Delta y_{4}$, and (3) the positions $y_{1}, y_{3}$ combined with the aileron spans $\Delta y_{2}, \Delta y_{4}$. Furthermore, the chord of the ailerons is not changed to maintain structural integrity of the wing as its spars need not be modified. Note that for optimization, aileron geometry constraints and parameter limits are introduced to avoid invalid configurations like overlaps or boundary violations (Fig. 4).

\subsection{Objective function}

Since the structural weight of a wing is largely driven by the maximum wing root bending moment (WRBM), the main goal for GLA controller design is to reduce the increments of the WRBM due to gust encounters $\Delta P_{\text {WRBM }}^{\text {gust }}$. Based on that, the objective function to be minimized is given as

$V=\max _{F} \max _{H} \Delta P_{\text {WRBM }}^{\text {gust }}$,

where $F$ and $H$ denote the considered discrete flight points and gust gradient distances, respectively. Hereinafter, the objective function $V$ is also referred to as performance index for GLA controller evaluation.

\subsection{Constraints}

\subsubsection{Limit loads}

While reducing structural loads at some parts of the aircraft, the GLA system might induce additional loads at other parts, e.g. at the CS mountings. Thus, it is necessary to ensure that the limit loads of the aircraft structure (see Sect. 2.4) are not exceeded at any cross-section. To that end, the constraint

$C_{1}: \mathbf{P}_{\mathrm{c} \text {,lower }} \leq \mathbf{P}_{\mathrm{c}} \leq \mathbf{P}_{\mathrm{c} \text {,upper }}$,

is introduced, where the $c$-set includes all relevant cut loads for aircraft sizing.

\subsubsection{Passenger comfort}

Furthermore, passenger comfort needs to be considered during GLA controller design. To that end, the comfort criterion $f_{c}$ from [18] is applied to the vertical acceleration measurement $a_{z \text {,meas }}$ of the IMU. The criterion is based on the ISO 2631-1 standard, which takes into account vibrating comfort and motion sickness phenomenon by weighting predefined frequencies. It is computed as the root mean square (RMS) of $a_{z \text {,meas }}$ weighted by the filter $W_{\mathrm{c}}(s)$ depicted in Fig. 5 . The evaluated comfort with active GLA should not be worse than a reference comfort determined by simulations without GLA. Hence,

$C_{2}: f_{\mathrm{c}}\left(a_{z \text {,meas }}\right) \leq f_{\mathrm{c}}\left(a_{z \text {,meas,ref }}\right)$.

Note that simulations without active GLA are independent of the CS configuration, as no deflections are applied and the mass distribution is assumed to be constant (see also Sect. 2.2).

\subsubsection{Stability}

As static gain feedback does not guarantee any stability, a stability analysis is carried out on the linearized closed loop model of the aircraft. The resulting minimum damping ratio $\zeta_{\min }$ is then compared to the reference value from the openloop case:

$C_{3}: \zeta_{\min } \geq \zeta_{\text {min,ref }}$

\subsubsection{Actuators}

To consider actuator limitations, the allowed CS deflections are constrained by

$C_{4}:\left\{\begin{array}{c}\eta_{\text {min }} \leq \eta \leq \eta_{\max } \\ \xi_{\text {inner,min }} \leq \xi_{\text {inner }} \leq \xi_{\text {inner,max }}, \\ \xi_{\text {outer,min }} \leq \xi_{\text {outer }} \leq \xi_{\text {outer,max }}\end{array}\right.$

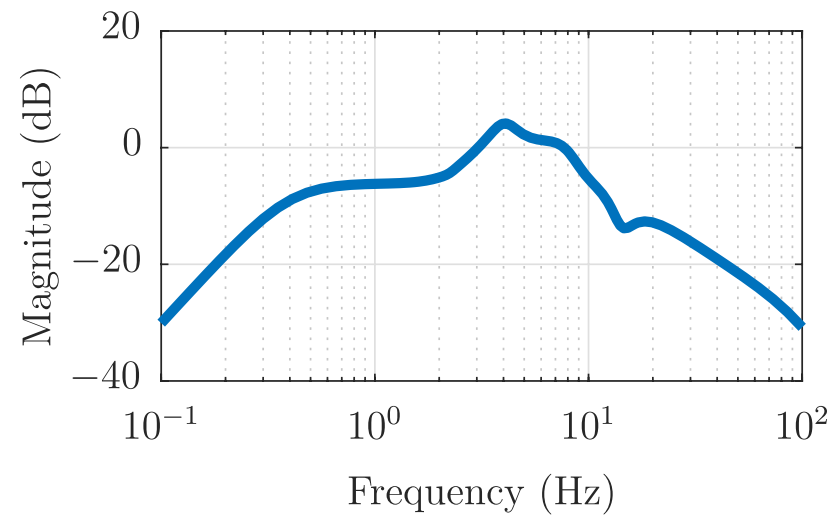

Fig. 5 Comfort weighting filter $W_{c}(s)$ 
where the CS deflection boundaries are obtained from the minimum and maximum deflections of the certification maneuvers listed in Sect. 2.4. This allows to define reasonable limitations depending on the current CS layout which limits the occurring hinge moments [19]. Furthermore, the deflection rates are limited by

$C_{5}:\left\{\begin{array}{l}\dot{\eta}_{\min } \leq \dot{\eta} \leq \dot{\eta}_{\max } \\ \dot{\xi}_{\text {inner,min }} \leq \dot{\xi}_{\text {inner }} \leq \dot{\xi}_{\text {inner,max }} \\ \dot{\xi}_{\text {outer,min }} \leq \dot{\xi}_{\text {outer }} \leq \dot{\xi}_{\text {outer,max }}\end{array}\right.$

with the maximum achievable deflection rate of all CSs being set to $80^{\circ} \mathrm{s}^{-1}$ in both directions.

\subsubsection{Handling qualities}

As ailerons are also used for lateral control of the aircraft, lateral maneuverability must be maintained. According to the certification requirements $[2,3]$ as well as the handling qualities requirements [20], roll performance is defined by the time a certain bank angle change can be accomplished. By defining an achievable roll rate of at least $15^{\circ} \mathrm{s}^{-1}$ (see also Sect. 2.4), these requirements are generally fulfilled, not considering any changes in the acceleration behavior. However, roll acceleration basically depends on actuator dynamics and mass moment of inertia [17], which are both assumed not to be affected when changing the control surface layout. Thus, no further handling quality constraints are introduced here.

\subsubsection{Rigid body motions}

Since the deflection of the ailerons for GLA induces a pitching moment, it is required to compensate the resulting pitching motion using the elevators. To enforce that, the pitch rate is constrained by

$C_{6}: q_{\min } \leq q \leq q_{\max }$,

where the maximum and minimum pitch rate are derived from simulations without GLA.

\subsection{Optimization problem formulation}

Finally, the overall aeroservoelastic optimization problem can be formulated as

$\min _{\mathbf{D}_{K}, \mathbf{D}_{\text {ail }}} V$ s.t. $C_{1} \cdots C_{6}$ are satisfied,

with the objective function $V$ from Sect. 3.3 and the constraints $C_{1} \cdots C_{6}$ defined in Sect. 3.4. The design variables are the controller tuners $\mathbf{D}_{K}$ from Sect. 3.1 and the aileron parameters $\mathbf{D}_{\text {ail }}$ defined in Sect. 3.2. The optimization is performed with MOPS [21] using a gradient based sequential quadratic programming (SQP) algorithm. In each optimization step, the limit loads (Sect. 2.4) of the current aircraft configuration without GLA are computed. Subsequently, the GLA controller is derived, and the objectives and constraints are evaluated with respect to the actual limit loads.

\section{Results and discussion}

For the following results, which are based on the findings of [22], one single flight point $F$ at an altitude $h=8297 \mathrm{~m}$ and a Mach number $M a=0.85$ is considered. The aircraft is assumed to be fully loaded with a minimum amount of fuel in the wings, which is the mass case yielding the largest wing loads during gust encounters. Furthermore, up- and downwards gusts with four different gust gradient distances $H=30 \mathrm{ft}, 150 \mathrm{ft}, 300 \mathrm{ft}$ and $350 \mathrm{ft}$ are evaluated in each optimization step. Additional flight points and gusts can be taken into account easily, but have been neglected to simplify result interpretation and to save computation time. Besides, the unsteady AIC matrix is computed at 8 frequency points, where the lifting surfaces are discretized by 3526 aerodynamic boxes, see also Fig. 6. Subsequently, the RFA is performed with a number of 6 predefined poles. Taking into account the first 40 flexible modes, this leads to a total number of 888 states for the nonlinear aircraft model.

To obtain satisfying optimization results, it has been found sufficient to consider the shear force, bending- and torsional-moment at three cross sections of the wing (including the wing root) and the root of the horizontal tail plane (HTP). Note that due to the symmetric excitation, the resulting loads and accelerations at the left- and right-hand side of the aircraft are identical and thus are only considered once. In summary, the optimization problem consists of 154 constraints and 10 tuning parameters.

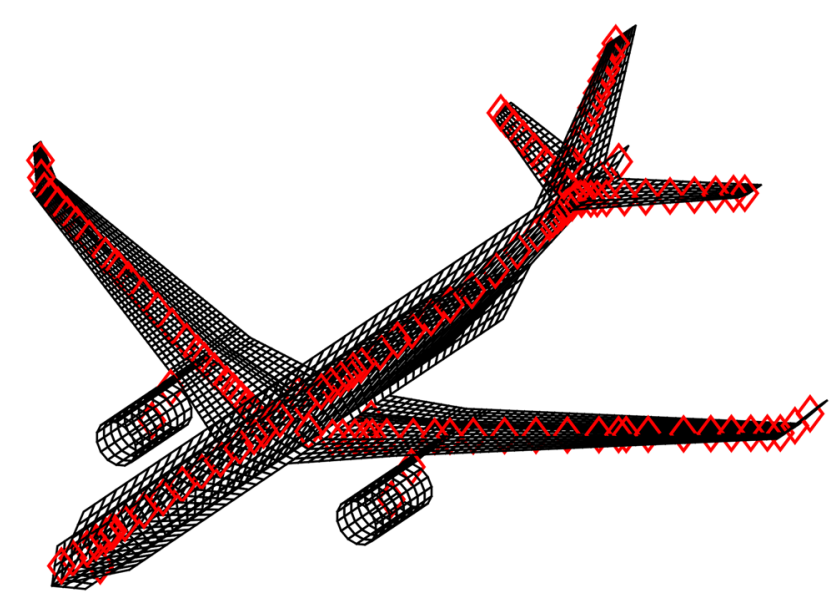

Fig. 6 Aircraft model with boxes of the aerodynamic model (black) and nodes of the condensed finite element model (red) 


\subsection{Comparison of optimization results}

First of all, a GLA system is tuned for the reference aileron configuration depicted in Fig. 7. To that end, the optimization problem defined in Eq. (6) is solved for a fixed set of aileron parameters $\mathbf{D}_{\text {ail }}$. The resulting reference controller reduces the WRBM increments forming the objective function (5) by $21 \%$. Second, geometry parameters of inner and outer ailerons are optimized simultaneously with controller gains. As a result, the maximum WRBM can be reduced in total by $30 \%$, which means that the GLA performance can be improved by $9 \%$ using the optimized aileron geometry depicted in Fig. 8. For both GLA systems, the gust gradient distance causing the largest WRBM is $300 \mathrm{ft}$, which also coincides well with Pratt's critical gust gradient distance of 12.5 reference chord lengths [23].

The respective time signals of the loads at the wing root are plotted in Fig. 9 for this critical gust, which is illustrated in the background. The reduction of the maximum WRBM can be clearly seen in Fig. 9. Additionally, the shear force is reduced as well, but the torsional moment is increased. Basically, the more the WRBM is reduced, the more the wing root torsional moment (WRTM) is increased due to the necessary aileron deflections. This is also depicted in Fig. 10, where the gust limit loads are compared over the whole wing. For a better comprehension, in the upper part of the two plots the reference aileron positions are shaded and in the lower part the optimized ones are shaded. Hence, the influence of the respective aileron layout on the torsional moment can be clearly recognized. In addition to that, the arising question of balancing the two different load channels is discussed in Sect. 4.3.

In Fig. 11, the required CS deflections for load alleviation are compared, where the deflection limits for both layouts are also marked. As already described in Sect. 3.4, the deflection limitations are determined from certification maneuvers and thus differ from each other for different aileron layouts. Generally, the deflection constraints, as well as the deflection rate bounds, are a major limiting factor for the achievable performance. However, it has to be noted that for the chosen controller structure, these limitations do not necessarily come to effect at critical gust gradient distances but rather at very short ones.

Furthermore, the control effectiveness for roll maneuvers can be directly determined for both control surface layouts by dividing the maximum roll rate $p_{\max }=15^{\circ}$ by the deflection limitations given in Fig. 11. Clearly, the optimized aileron layout provides a greater control effectiveness because the overall control surface size is larger. The given control effectiveness is a sum of the effectiveness of the individual pairs of control surfaces as it can be seen in Table 2 . The outer ailerons are less effective, not only because they are smaller but also because corresponding deflections yield

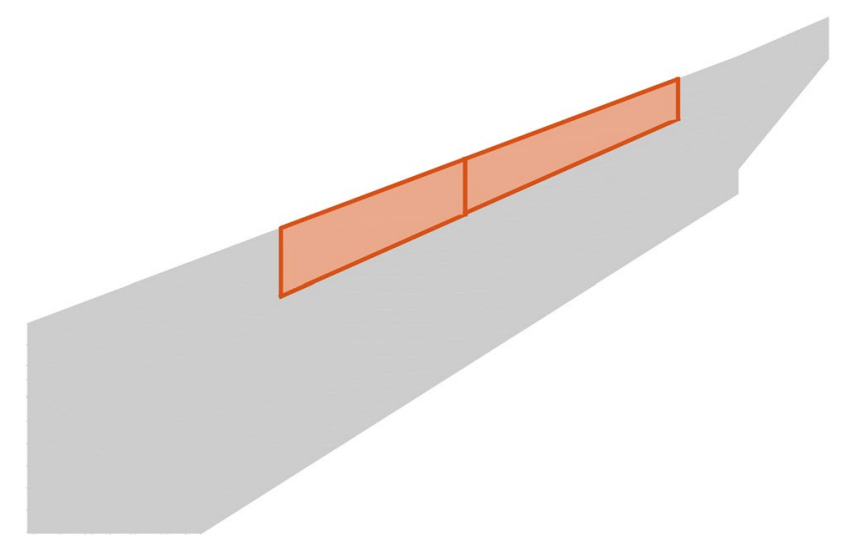

Fig. 7 Reference aileron layout

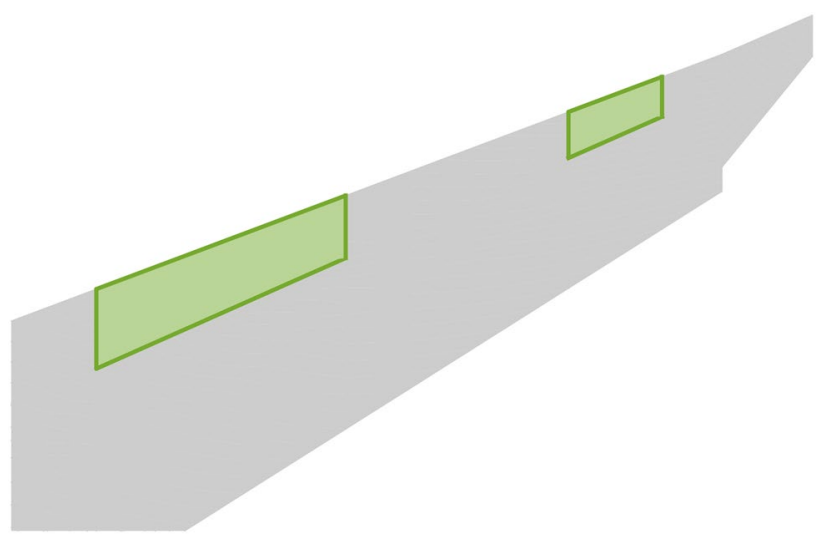

Fig. 8 Optimized aileron layout

a larger torsion of the outer wing. Note that at higher Mach numbers, the control effectiveness can even become negative which is also known as control reversal [24].

\subsection{Discussion of the optimized aileron layout}

By varying the initial values of the controller tuners or the aileron layout, different results with similar objective values are obtained. This means, the solution is not unique, giving additional degrees of freedom to the engineer. However, it appears that the inner ailerons are always placed similarly as depicted in Fig. 8, whereas the position and span of the outer ailerons seem to have a minor influence on GLA performance. In order to find an explanation for this result, a closer look is taken on the modal displacements leading to the maximum WRBM occurring at $t \approx 0.6 \mathrm{~s}$, see Fig. 9. To that end, the contributions of each flexible mode to the WRBM are computed according to the MDM described in Eq. (4). The contributions are normalized by the maximum occurring WRBM without GLA, and displayed in Table 3, where only the modes 


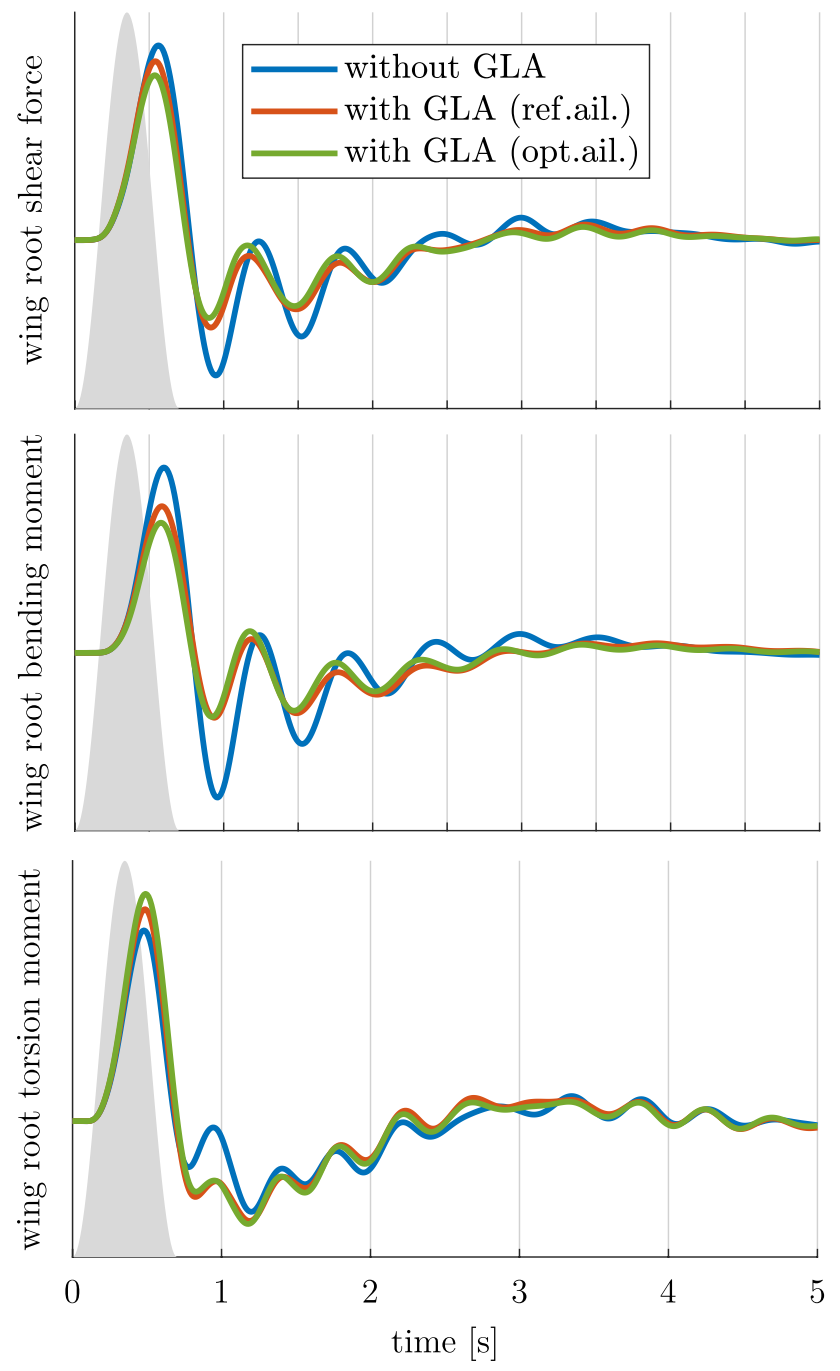

Fig. 9 Loads at wing root for critical gust

with the highest impact are listed. For the sake of clarity, summing up the normalized WRBM contributions of all flexible modes would lead to the performance index $V$ of the respective aircraft configuration. It can be seen that the WRBM is clearly dominated by the first symmetric wing bending mode (mode 1). Hence, the GLA system should primarily damp this mode without exciting any other modes, which is assumed to be crucial when using the reference aileron layout for GLA. Comparing the first two rows of Table 3 , it is shown that the contributions of the modes 10, 12 and 21 are increased when using the reference aileron configuration. In contrast, using the optimized ailerons for GLA, modes 10 and 12 are damped instead of excited. The reason for that can be seen in Fig. 12, where the vertical wing displacements for the corresponding mode shapes are shown for the maximum WRBM at $t \approx 0.6 \mathrm{~s}$. Again, in the upper part of the plot, the positions of the reference ailerons are marked, and

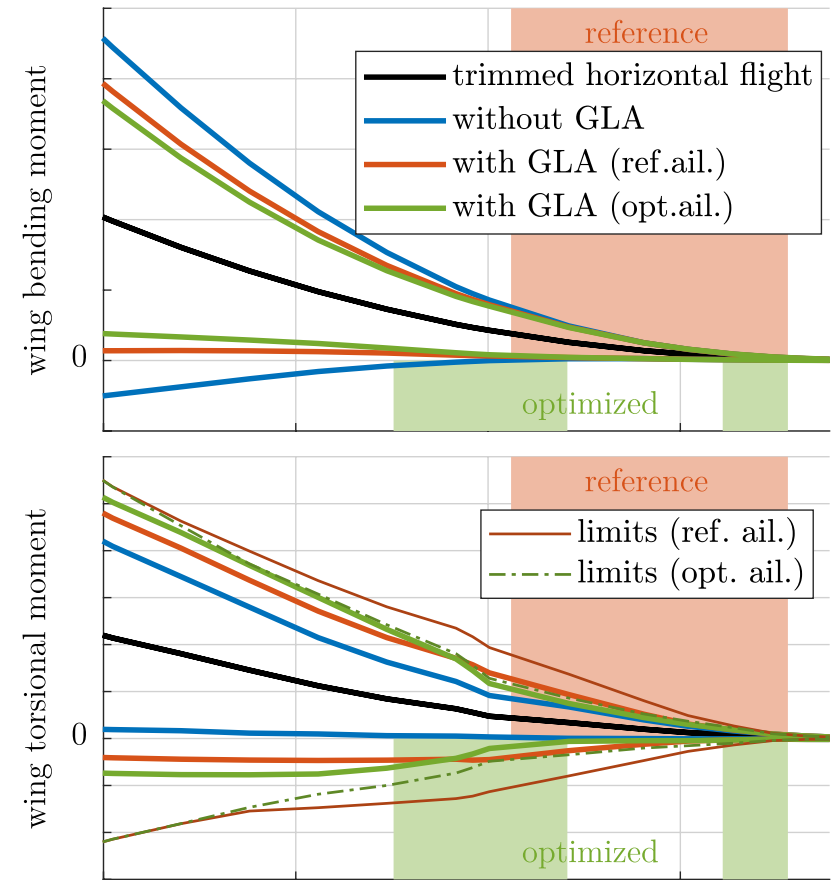

right wing inner $\rightarrow$ outer

Fig. 10 Bending and torsional moment on the wing for the considered "1-cos" gusts
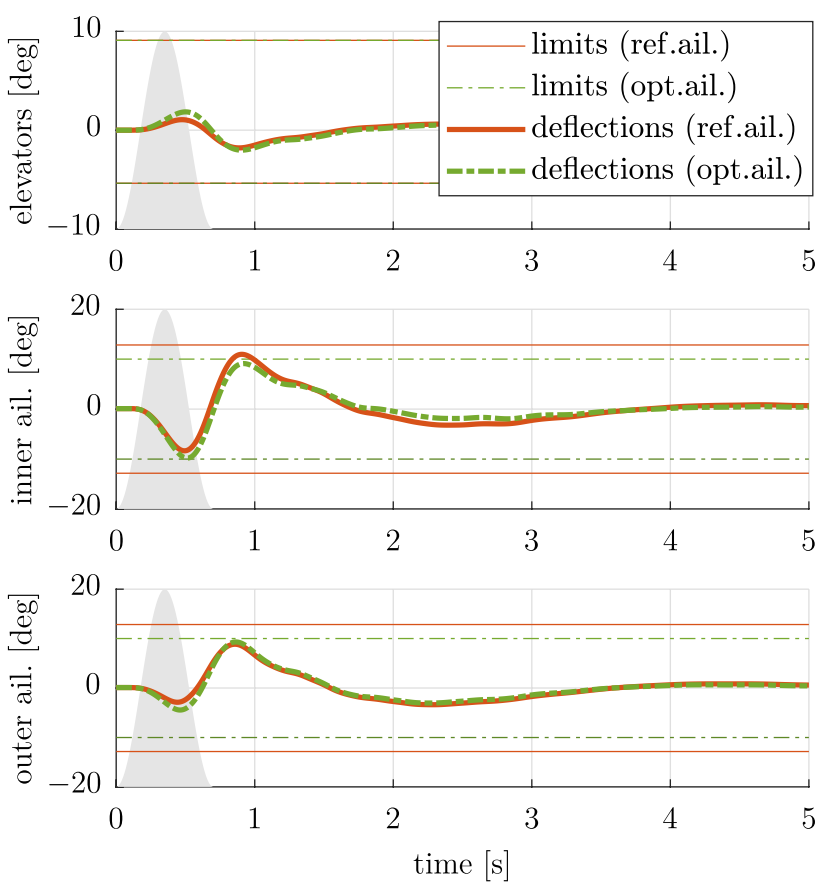

Fig. 11 CS deflections for critical gust

in the lower part, the positions of the optimized ailerons are marked. The mode shapes 10 and 12 appear to be very similar for this mass case and it can be seen that the 
Table 2 Aileron effectiveness for rolling

\begin{tabular}{lll}
\hline Ailerons & $\begin{array}{l}\text { Refer- } \\
\text { ence } \\
\text { layout }\end{array}$ & $\begin{array}{l}\text { Opti- } \\
\text { mized } \\
\text { layout }\end{array}$ \\
\hline Inner only & 0.8 & 1.4 \\
Outer only & 0.4 & 0.1 \\
Both & 1.2 & 1.5 \\
\hline
\end{tabular}

optimized inner ailerons are placed further inward than the respective oscillation node. Hence, the vertical displacements of modes 1,10 and 12 point in the same direction at the range of the inner ailerons. For this reason, a coordinated deflection of the optimized inner ailerons allows damping all three modes simultaneously at this instant of time. Furthermore, the undesired excitation of mode 21 indicates that a compromise is made for the optimal placement of the ailerons. Note that this interpretation is not unambiguous as, for instance, the solution of the optimization problem also depends on many different constraints given in Sect. 3.4.

\subsection{Load balancing}

As already mentioned, actively reducing the wing bending moment is at the cost of an increased wing torsional moment. In addition to that, the loads at the HTP are increased as well due to the deflections of the elevators for pitching moment compensation. This can also be seen in Fig. 13a, b, where the correlated gust loads of the wing root and the HTP root are compared, respectively. A tradeoff study is carried out to identify the Pareto front between the WRBM and the WRTM. To that end, the allowable WRTM is successively reduced and the respective achievable GLA performance is determined. As depicted in Fig. 14, this results in a monotonic decrease of the GLA performance for both the fixed reference aileron configuration and a variable aileron configuration to be optimized. In case the closed-loop WRTM is limited to the open-loop WRTM (no GLA), an active alleviation of the WRBM is not possible, even if the aileron layout is optimized. Furthermore, not limiting the WRTM at all does not lead to any better performance than already presented above. Interestingly, setting the WRTM limits to the values from the reference GLA system but allowing an optimization of
Table 3 Comparison of modal contributions to maximum WRBM

\begin{tabular}{lrllll}
\hline Aircraft configuration & $V(\%)$ & Mode 1 $(\%)$ & Mode 10 $(\%)$ & Mode 12 (\%) & Mode 21 (\%) \\
\hline Without GLA & 100 & 93.79 & 2.72 & 1.36 & 0.82 \\
With GLA (reference ailerons) & 79 & 69.78 & 3.54 & 1.7 & 2.07 \\
With GLA (optimized ailerons) & 70 & 61.79 & 2.53 & 0.42 & 3.53 \\
\hline
\end{tabular}

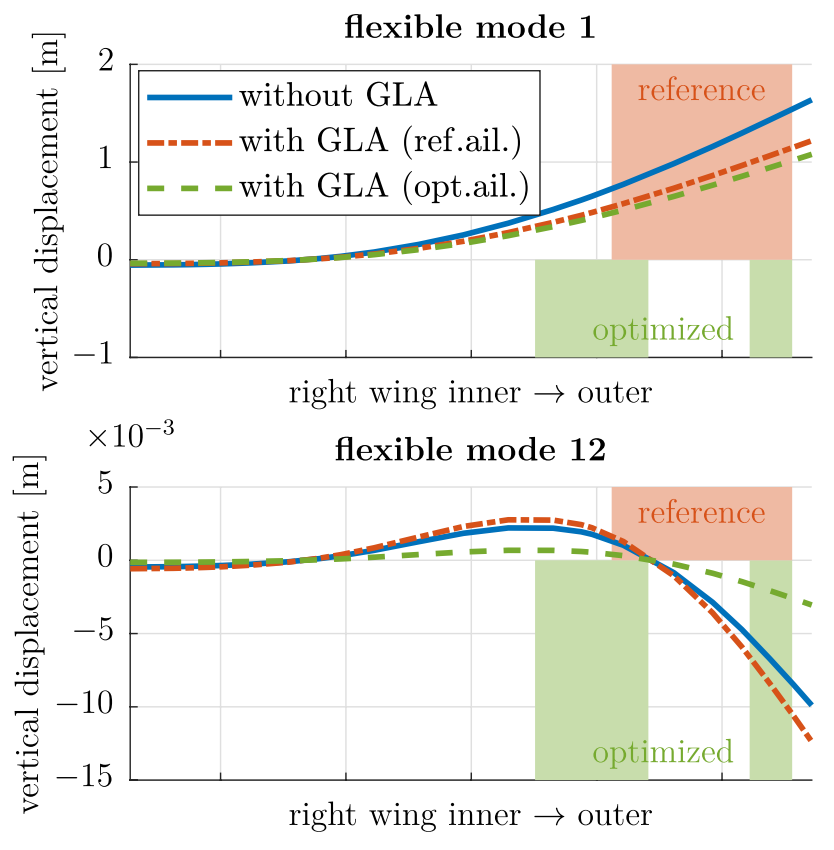

Fig. 12 Comparison of modal displacements for maximum WRBM

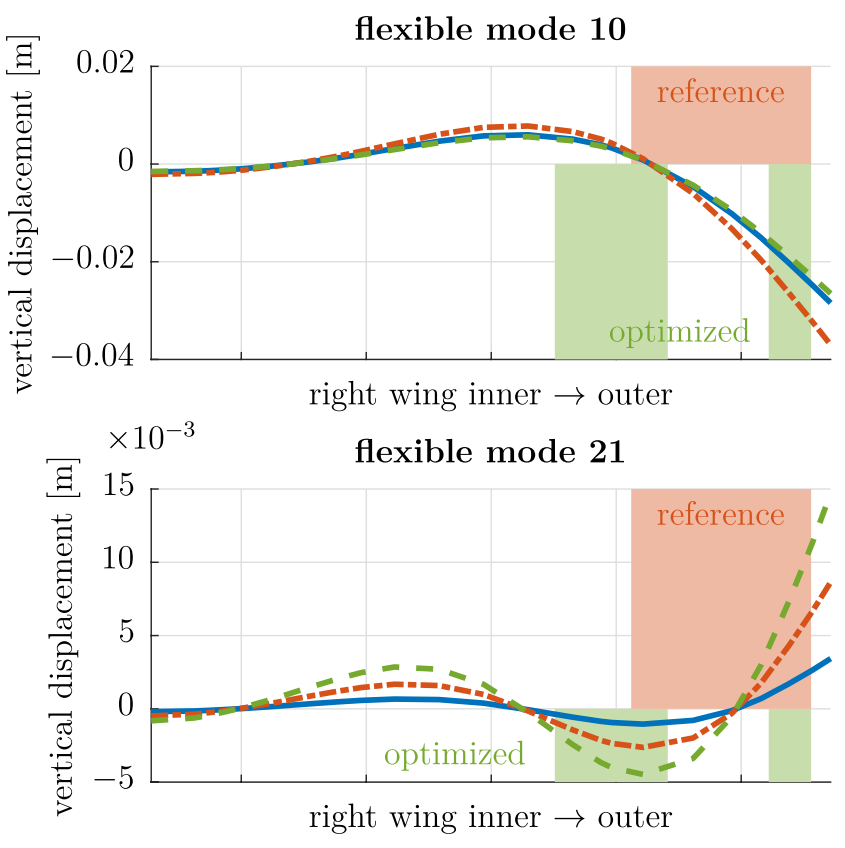




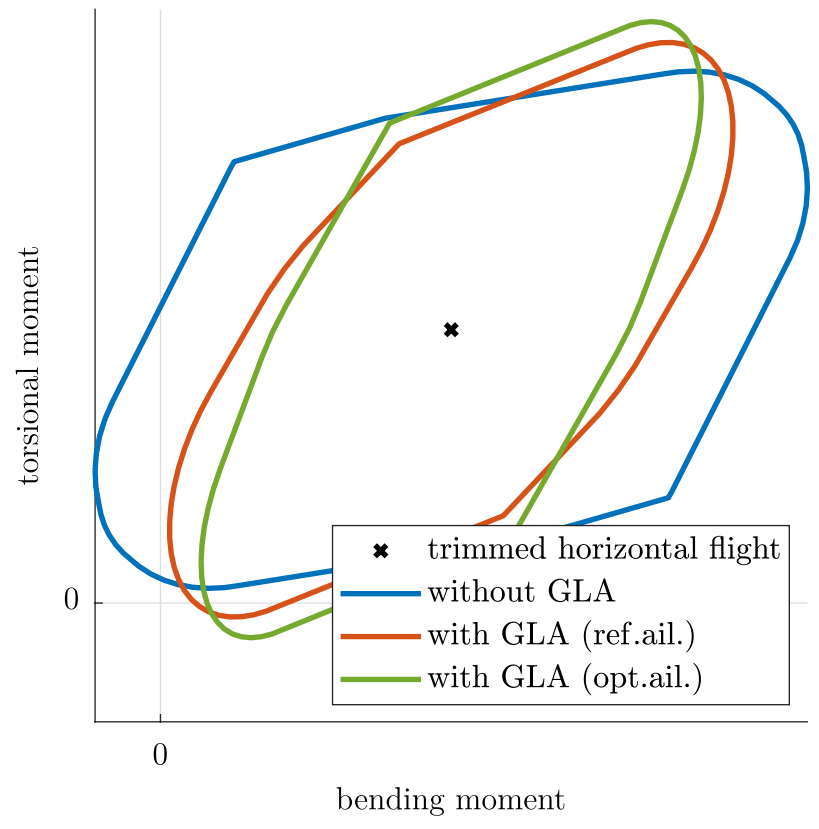

(a) Correlated gust loads at wing root.

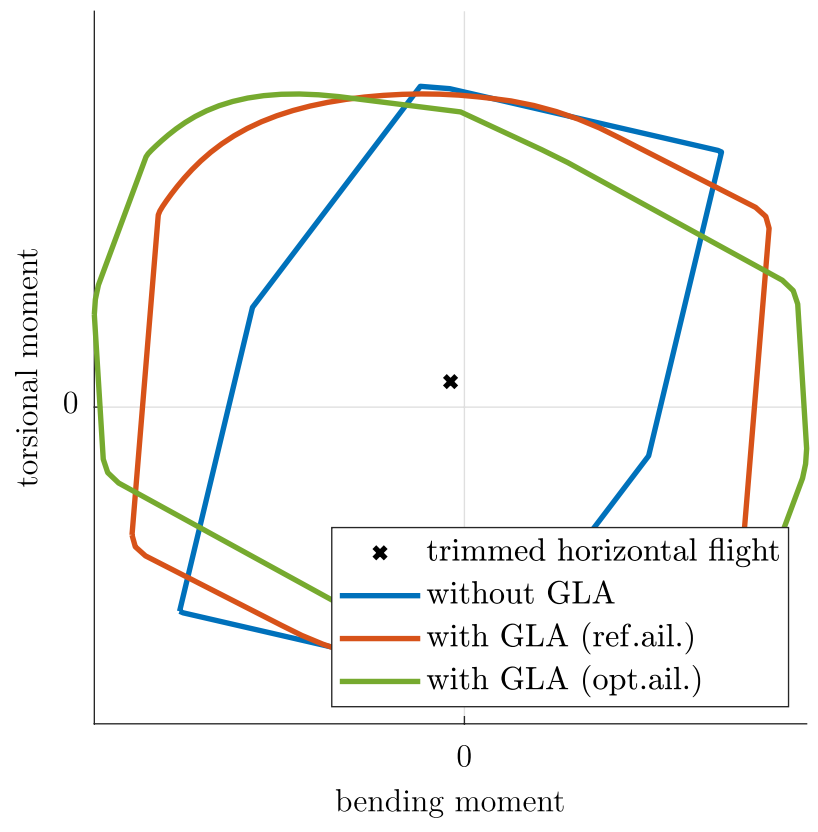

(b) Correlated gust loads at HTP root.

Fig. 13 Comparison of correlated gust loads

the aileron layout does not lead to an improvement of the GLA performance. This means that the reference aileron configuration is already optimal if no further increase of the WRTM is allowed.

Similarly, limiting the bending moment at the root of the HTP leads to a decrease of the achievable GLA performance (not depicted). Nevertheless, selecting the WRBM as the overall GLA objective provides a higher potential for structural weight savings than reducing the WRTM or the loads at the HTP as discussed in Sect. 3.3. However, any other trade-off point may be selected taking into account further engineering aspects.

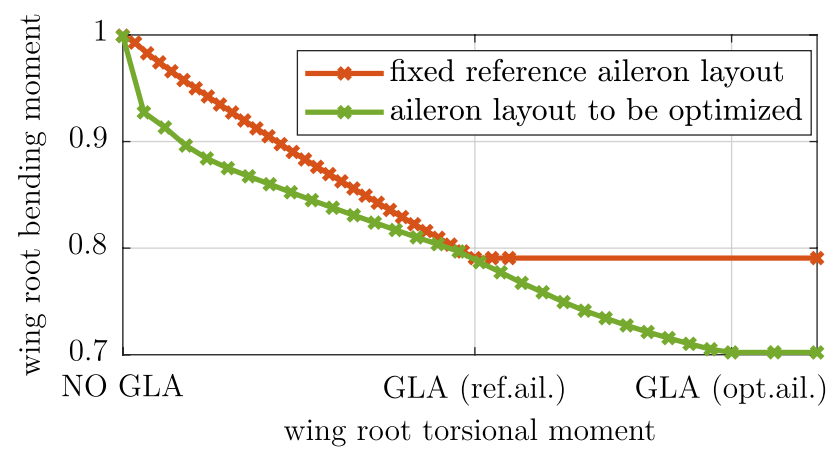

Fig. 14 Comparison of achievable GLA performance depending on WRTM limitations

\section{Conclusion and outlook}

The aeroservoelastic optimization framework presented in this paper allows to simultaneously tune the controller and the control surface (CS) layout for the purpose of active (GLA). An efficient update routine for changes of the nonlinear aircraft model with unsteady aerodynamics is introduced. To obtain a reasonable solution, multiple constraints are introduced including limitations of loads at different cross sections, actuator bandwidth and passenger comfort. The resulting GLA system with an optimized aileron geometry allows to reduce the wing root bending moment (WRBM) by $30 \%$, whereas with the reference aileron configuration only $21 \%$ can be achieved. An active reduction of the WRBM leads to an increase of the wing root torsional moment (WRTM) and the horizontal tail plane (HTP) loads, and thus, a trade-off has to be made. On the basis of individual mode shapes, the optimal placement of the ailerons is explained, where the dependency on the actual mass case needs to be considered. For future investigations, it is necessary to take into account the whole design envelope, which increases the complexity of the optimization problem. In addition to that, the interaction of the GLA system with the electronic flight control system (EFCS) also has to be considered. Apart from that, further performance improvements are expected if a more advanced controller structure or additional CSs like spoilers are used. Last but not least, the concrete weight savings need to be determined to evaluate the impact on the direct operating costs of the aircraft. 
Acknowledgements Part of the research has been funded in the course of the European Union's Seventh Framework Program (FP7/20072013) in the Clean Sky Joint Technology Initiative under Grant agreement CSJU-GAM-SFWA-2008-001.

Open Access This article is distributed under the terms of the Creative Commons Attribution 4.0 International License (http://creativeco mmons.org/licenses/by/4.0/), which permits unrestricted use, distribution, and reproduction in any medium, provided you give appropriate credit to the original author(s) and the source, provide a link to the Creative Commons license, and indicate if changes were made.

\section{References}

1. International Energy Agency: Transport, Energy and $\mathrm{CO}_{2}$. IEA Publication, Paris, France (2009)

2. Federal Aviation Administration: Federal Aviation Regulations Part 25. Transport Category, Airworthiness Standards (2015)

3. Agency, European Aviation Safety: Certification Specifications and Acceptable Means of Compliance for Large Aeroplanes, CS-25. Amendment 16 (2015)

4. Johnston, J.: Accelerated development and flight evaluation of active controls concepts for subsonic transport aircraft. Volume 1: load alleviation/extended span development and flight tests. NASA (1979)

5. Regan, C.D., Jutte, C.V.: Survey of applications of active control technology for gust alleviation and new challenges for lighterweight aircraft. NASA (2012)

6. Flaig, A.: Airbus A380: solutions to the aerodynamic challenges of designing the worlds largest passenger aircraft. Royal Aeronautical Society Hamburg Branch Lecture Series (2008)

7. Kaminski-Morrow, D.: Airbus exploits A320 load-alleviation to offer higher MTOW. Flight Global (2008). http://www.flightglob al.com/news/articles/airbus-exploits-a320-load-alleviation-tooff er-higher-mtow-319049/

8. Livne, E.: Integrated aeroservoelastic optimization: status and direction. J. Aircr. 36(1), 122-145 (1999). https://doi. org/10.2514/2.2419

9. Haghighat, S., Martins, J.R.R.A., Liu, H.H.T.: Aeroservoelastic design optimization of a flexible wing. J. Aircr. 49(2), 432-443 (2012)
10. Xu, J., Kroo, I.: Aircraft design with active load alleviation and natural laminar flow. J. Aircr. 51(5), 1532-1545 (2014)

11. Kier, T., Looye, G.: Unifying manoeuvre and gust loads analysis models. In: International Forum on Aeroelasticity and Dynamics. Seattle (2009)

12. Waszak, M.R., Schmidt, D.K.: Flight dynamics of aeroelastic vehicles. J. Aircr. 25(6), 563-571 (1988)

13. Rodden, W., Johnson, E.: MSC.Nastran Version 68, Aeroelastic Analysis and User's Guide (2004)

14. Roger, K.L.: Airplane math modelling methods for active control design. Structures and Materials Panel (1977)

15. Bisplinghoff, R., Ashley, H., Halfman, R.: Aeroelasticity. Dover Publications, Mineola, NY, USA (1955)

16. Fezans, N., Joos, H.D.: Combined feedback and lidar-based feedforward active load alleviation. In: AIAA Atmospheric Flight Mechanics Conference, p. 3548 (2017)

17. Sadraey, M.H.: Aircraft Design: A Systems Engineering Approach. Wiley, Chichester, UK (2012)

18. Kubica, F., Madelaine, B.: Passenger comfort improvement by integrated control law design. Tech. rep, DTIC Document (2000)

19. Hoblit, F.M.: Gust loads on aircraft: concepts and applications. AIAA (1988)

20. Moorhouse, D., Woodcock, R.: US Military Specification MILF-8785C (1980)

21. Joos, H.D.: A multiobjective optimisation-based software environment for control systems design. In: Computer Aided Control System Design, 2002. Proceedings. 2002 IEEE International Symposium, pp. 7-14. IEEE (2002)

22. Pusch, M., Knoblach, A., Kier, T.: Integrated optimization of ailerons for active gust load alleviation. In: International Forum on Aeroelasticity and Structural Dynamics, Saint Petersburg, Russia, paper, p. 199 (2015)

23. Pratt, K.G., Walker, W.G.: A revised gust-load formula and a reevaluation of $\mathrm{v}$-g data taken on civil transport airplanes from 1933 to 1950. Tech. rep., NACA Report No. 1206 (1954)

24. Schmidt, D.K.: Modern flight dynamics. McGraw-Hill, New York (2012)

Publisher's Note Springer Nature remains neutral with regard to jurisdictional claims in published maps and institutional affiliations. 